\title{
Hybrid SSB OFDM-Digital Filter Multiple Access PONs
}

Jin, Wei; Sankoh, Abdulai; Dong, Yixian; Giddings, Roger; O'Sullivan, M.; Durrant, T.; Lee, J.; Tang, Jianming

\section{Journal of Lightwave Technology}

DOI:

https://doi.org/10.1109/JLT.2020.2966287

Published: 15/04/2020

Peer reviewed version

Cyswllt i'r cyhoeddiad / Link to publication

Dyfyniad o'r fersiwn a gyhoeddwyd / Citation for published version (APA):

Jin, W., Sankoh, A., Dong, Y., Giddings, R., O'Sullivan, M., Durrant, T., Lee, J., \& Tang, J. (2020). Hybrid SSB OFDM-Digital Filter Multiple Access PONs. Journal of Lightwave Technology, 38(8), 2095 - 2105. https://doi.org/10.1109/JLT.2020.2966287

\footnotetext{
Hawliau Cyffredinol / General rights

Copyright and moral rights for the publications made accessible in the public portal are retained by the authors and/or other copyright owners and it is a condition of accessing publications that users recognise and abide by the legal requirements associated with these rights.

- Users may download and print one copy of any publication from the public portal for the purpose of private study or research.

- You may not further distribute the material or use it for any profit-making activity or commercial gain

- You may freely distribute the URL identifying the publication in the public portal ?
}

Take down policy

If you believe that this document breaches copyright please contact us providing details, and we will remove access to the work immediately and investigate your claim. 


\title{
Hybrid SSB OFDM-Digital Filter Multiple Access PONs
}

\author{
W. Jin, A. Sankoh, Y.X. Dong, Z.Q. Zhong, R.P. Giddings, Member, IEEE, M. O’Sullivan, J. Lee, T.
} Durrant and J.M. Tang Member, IEEE

1

Abstract- The previously reported hybrid orthogonal frequency division multiplexing-digital filter multiple access (OFDM-DFMA) PONs offer promising solutions for seamlessly converging optical and mobile networks for 5G. However, the hybrid OFDM-DFMA PONs based on intensity-modulation and direct-detection (IMDD) convey double sideband (DSB) signals at different sub-wavelengths, this halves the spectral efficiency and signal transmission capacity. In this paper, hybrid single sideband (SSB) OFDM-DFMA PONs are proposed and explored, for the first time, where multiple SSB OFDM channels produced without employing the Hilbert transform operation are multiplexed using orthogonal digital filtering in ONUs, and demultiplexed/demodulated by a single FFT operation in OLT without incorporating matching filters. It is shown that similar to the DSB PON, the proposed SSB PON has excellent robustness against digital filter characteristic variations and channel interferences. More importantly, it decreases peak-to-average power ratios (PAPRs) of digitally-filtered OFDM signals by >2dB, thus leading to $>2 \mathrm{~dB}$ reductions in optimum signal clipping ratio and $>1$ bit reductions in minimum required DAC/ADC resolution bits. For fixed spectral bandwidths, the SSB PON almost doubles the maximum upstream transmission capacity without considerably degrading its differential ONU optical launch power dynamic range compared to the DSB PON. While for fixed upstream signal transmission capacities, compared to the DSB PON, the SSB PON not only halves the overall signal transmission bandwidth, but also leads to $>2.5 \mathrm{~dB}(>1.2 \mathrm{~dB})$ improvements in power budget (differential optical launch power dynamic range for ONUs locating at high frequency regions).

Index Terms - Digital orthogonal filtering, single sideband (SSB), intensity modulation and direct detection (IMDD).

\section{INTRODUCTION}

$\mathrm{T}$

o meet the high bandwidth, low latency and ultra-dense elastic connection requirements for $5 \mathrm{G}$ and beyond networks, cloud access networks (CANs) have been proposed to seamlessly converge optical access networks, metropolitan area networks and mobile front-haul/back-haul networks [1]. To practically implement CANs, an intensity modulation and direct detection (IMDD)-based passive optical network (PON)

This work was supported by the DESTINI project funded by the ERDF under the SMARTExpertise scheme.

W. Jin, A. Sankoh, Y.X. Dong, Z.Q. Zhong, R.P. Giddings and J.M. Tang are with the School of Electronic Engineering, Bangor University, Bangor, LL57 1UT, UK. (email: w.jin@bangor.ac.uk ; j.tang@bangor.ac.uk).

M. O'Sullivan is with Ciena Canada, Inc., 385 Terry Fox Drive, Ottawa, Ontario K2K 0L1, Canada.

J. Lee and T. Durrant are with EFFECT Photonics LTD. Brixham Laboratory, Freshwater Quarry, Brixham, Devon, England, TQ5 8BA, UK. is widely considered to be a promising candidate due to its excellent cost-effectiveness and power- efficiency [2], [3]. On the other hand, to fully accommodate the explosive expansion of various $5 \mathrm{G}$ and beyond mobile traffic having a wide diversity of characteristics, software defined networking (SDN) with its network control functionalities further extended to physical layer is also highly desirable for dynamically providing elastic network slicing, adaptive multicarrier modulation, simple but efficient network management, optimum network resource utilization as well as dynamic on-demand connections/services.

To address the aforementioned technical challenges, a digital filter multiple access (DFMA) PON has been proposed [4], which utilizes SDN-controllable digital orthogonal filters to dynamically and independently multiplex and demultiplex multiple channels of arbitrary bandwidth granularity without requiring expensive analogue optical/electrical components. Extensive theoretical investigations and experimental demonstrations of the DFMA PON have been conducted in the cost-effective IMDD application scenarios [4]-[6]. However, the number of required digital filtering process implemented in the optical line terminal (OLT)-embedded digital signal processing (DSP) function is proportional to optical network unit (ONU) count, therefore a large number of ONUs simultaneously accommodated by the PON can unavoidably result in relatively high OLT DSP complexity and operational expenditure.

To solve this technical issue, very recently, a hybrid orthogonal frequency division multiplexing (OFDM)-DFMA PON based on IMDD has been proposed and extensively explored [7], [8], where for upstream transmissions, individual ONUs use their embedded digital shaping filters (SFs) to locate their produced OFDM signals at different sub-wavelength spectral regions by applying following the operating principle similar to the DFMA PONs. While in the OLT, a single fast Fourier transform (FFT) operation and the following data recovery DSP processes are implemented in a pipelined way to simultaneously demultiplex and recover the signals from various ONUs without utilizing parallel matching filters (MFs). In comparison with the DFMA PONs, the hybrid OFDM-DFMA PONs have the following salient features, including [7]: 1) considerable relaxation of the stringent requirements on high complexity of the ONU transmitter-embedded SFs due to the enhanced system robustness against digital filter characteristic variations; 2) great simplification of the OLT DSP complexity and significant 


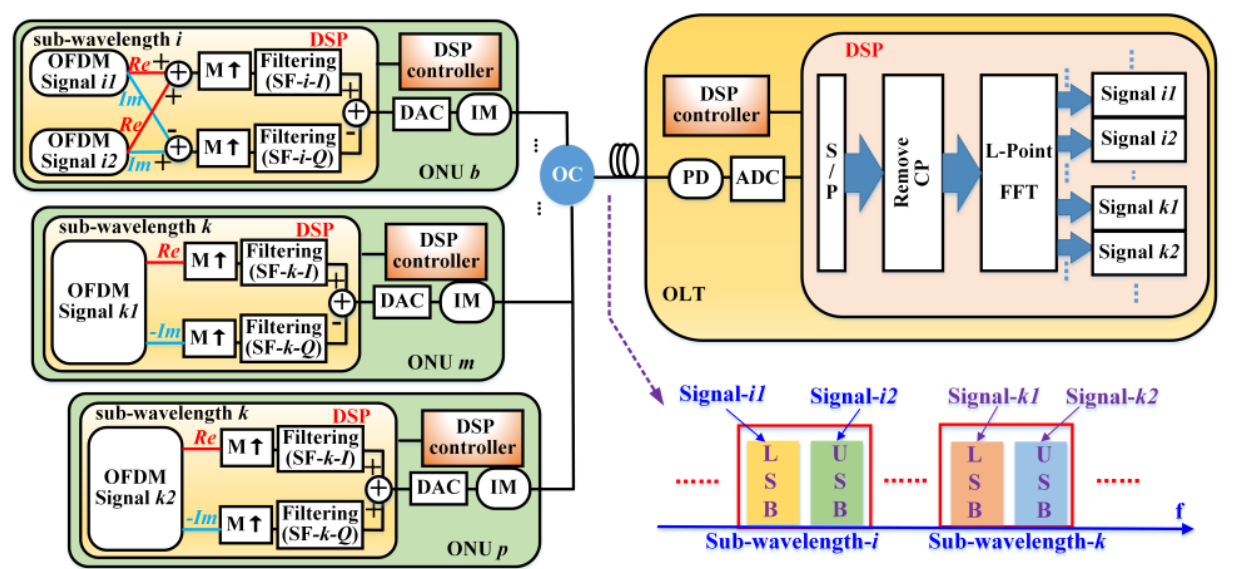

Fig. 1 Schematic diagram of the proposed IMDD hybrid SSB OFDM-DFMA PON without either performing the Hilbert transform operation or utilizing matching filters. SF: shaping filter. I/Q: in-phase/quadrature-phase digital filter. $\mathrm{M} \uparrow$ : digital-domain up-sampling operation by a factor of M. DAC/ADC: digital-to-analogue/analogue-to-digital conversion. IM: intensity modulation. PD: photodetector. S/P: serial-to-parallel conversion. CP: cyclic prefix. FFT: fast Fourier transform. LSB/USB: lower/upper sideband.

reductions in OLT expenditure and power consumption due to both the elimination of the MFs and the adopted pipelined signal recovery process; 3 ) considerable improvements in both upstream power budget and upstream performance robustness against practical transceiver/system impairments because of the improved system tolerance to multiple channel interferences and signal distortions, and 4) inherent transparency to the existing OFDM-based 4G networks.

However, in the previously reported hybrid OFDM-DFMA PONs, each individual sub-wavelength just conveys a single digitally-filtered double sideband (DSB) OFDM signal. As Tthe lower sideband (LSB) and the upper sideband (USB) of thea digitally-filtered DSB OFDM signal convey identical data information at different radio frequencies (RFs), it - It therefore leads to an almost halved spectral efficiency and a reduction in signal transmission capacity compared to the DFMA PONs [4].

To effectively address the above disadvantages and still maintain the aforementioned salient features associated with the previously-_reported hybrid DSB OFDM-DFMA PONs, in this paper, for IMDD application scenarios, a novel PON architecture termed hybrid single sideband (SSB) OFDM-DFMA PON is proposed and extensively investigated, for the first time, where in the upstream, without implementing the sophisticated Hilbert transform DSP operation, a complex SSB OFDM signal generation process and software-reconfigurable digital orthogonal filtering, as demonstrated in Section II, are utilized in each ONU. Each individual ONU can occupy a single spectral sideband or two independent spectral sidebands within the same sub-wavelength spectral region. In the OLT, for demultiplexing and demodulating the SSB OFDM signals from various ONUs, very similar to the previously reported hybrid DSB OFDM-DFMA PON, a single FFT operation and its following signal recovery processes are performed in a pipelined way without either utilizing digital MFs or implementing the Hilbert transform DSP operation. The elimination of the conventional Hilbert transform DSP operation in both the ONUs and the OLT can considerably decrease the transceiver DSP complexity.

Our results indicate that similar to the hybrid DSB OFDM-DFMA PONs, the proposed hybrid SSB OFDM-DFMA PONs can still offer numerous salient advantages including: 1) excellent robustness against digital filter characteristic variations; 2) ONU count-independent negligible channel interferences when adopting optimum digital filter length, and 3) inherent transparency to the $4 \mathrm{G}$ networks. In addition to the aforementioned advantages, in comparisons with the DSB cases, the proposed hybrid SSB OFDM-DFMA PON also possesses the unique features listed below:

1. $>2 \mathrm{~dB}$ reductions in the peak to average power ratios (PAPRs) of the digitally-filtered signals. Such PAPR reductions are independent of the digital filter characteristics and modulation formats as well as signal spectral locations. The reduced PAPRs can also lead to $>2 \mathrm{~dB}$ reductions in optimum signal clipping ratio.

2. >1-bit reductions in minimum required digital-to-analogue conversion/analogue-to-digital conversion (DAC/ADC) resolution bits for achieving bit error rates (BERs) of $1 \times 10^{-3}$. Such resolution reductions are mainly because of the SSB technique-induced decreases in PAPRs of the digitallyfiltered OFDM signals.

3. When the SSB and DSB cases have identical overall signal transmission bandwidths, A almost doubled maximum aggregated upstream signal transmission capacity and spectral efficiency are obtainable, which are similar to those offered by the standard complex

Formatted: Underline
Formatted: Underline
Formatted: Underline
Formatted: Underline




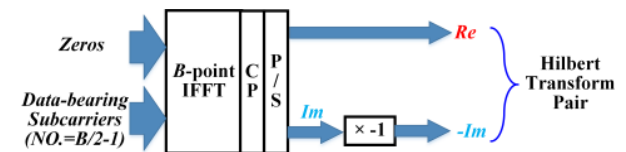

Fig. 2 Complex SSB OFDM signal generation process without utilizing the Hilbert transform operation. The real part $(R e)$ and (-1)-multiplied imaginary part (-Im) of the produced OFDM signal form a Hilbert transform pair. CP: cyclic prefix. P/S: parallel-to- serial conversion.

IFFT for fixed overall signal transmission bandwidth for the SSB and DSB cases. In addition, such a significant improvements in upstream signal transmission capacity and spectral efficiency does not do not affect the achievable ONU optical launch power dynamic ranges, i.e., there exist almost identical ONU optical launch power dynamic ranges for the above-mentioned two PON architectures.

4. For delivering similar upstream signal transmission capacity between two considered PON architectures, a halved overall signal transmission bandwidth and a $>2.5 \mathrm{~dB}$ upstream system power budget improvement are achievable. Moreover, $>1.2 \mathrm{~dB}$ improvements in differential optical launch power dynamic range are also observed for the ONUs locating at high frequency spectral regions. The halved overall bandwidth requirement allows the utilization of low-cost and low-bandwidth optical/electrical components, thus reducing the overall transceiver costs.

\section{THEORETICAL MODEL OF IMDD HYBRID SSB OFDM-DFMA PONS}

The proposed hybrid SSB OFDM-DFMA PON is illustrated in Fig. 1, in which the more challenging multipoint-to-point upstream operation is considered only. In the hybrid SSB OFDM-DFMA PON, by performing twe similar digital filtering processes, each ONU can produce either two different SSB OFDM signals occupying a single sub-wavelength spectral region or a single SSB OFDM signal occupying either a LSB or USB spectral region. In the OLT, a single $L$-point FFT operation is performed to demultiplex and demodulate the multiple SSB OFDM signals from various ONUs without utilizing MFs. Such a MF-free, pipelined OLT DSP procedure is similar to that applied in the previously reported hybrid DSB OFDM-DFMA PONs

In the ONUs, the complex SSB OFDM signal generation process illustrated in Fig. 2 is utilized to generate the SSB OFDM signals. As seen in this figure, assuming that the inverse fast Fourier transform (IFFT) size is $B$, the $m$-th OFDM symbol at the IFFT output can be expressed as,

$$
\begin{aligned}
& x_{m}(n)=\sum_{c=-\frac{B}{2}}^{\frac{B}{2}-1} d_{c, m} e^{j \frac{2 \pi c(n-m B)}{B}}, m=0,1,2 \ldots . \\
& d_{c, m}=\left\{\begin{array}{cc}
a_{c, m} & -\frac{B}{2}+1 \leq c \leq-1 \\
0 & \text { others }
\end{array}\right.
\end{aligned}
$$

where $a_{c, m}$ is the encoded information sample taken by the $c$-th subcarrier of the $m$-th OFDM symbol. The produced complex OFDM signal contains a total of $B / 2-1$ data-bearing subcarriers.

After adding the cyclic prefix (CP) and implementing the parallel-to-serial (P/S) conversion, the real part and (-1)-multiplied imaginary part of the produced complex OFDM signal $S(t)$ form a Hilbert transform pair [9], which satisfy the following relationship,

$$
\operatorname{Re}(S(t))=H\{-\operatorname{Im}(S(t))\}
$$

where $\operatorname{Re}(S(t))$ and $\operatorname{Im}(S(t))$ denote the real part and the imaginary part of the signal $S(t)$ respectively. $H\{\bullet\}$ stands for the Hilbert transform operation [10]. It is also worth mentioning that the real part of the produced complex OFDM signal $\operatorname{Re}(S(t))$ is similar to the corresponding real-valued OFDM signal generated by the Hermitian symmetry in the conventional IMDD hybrid DSB OFDM-DFMA PONs [7]. Therefore, such a signal generation process offers a simple but effective approach of simultaneously producing the required real-valued OFDM signal and its Hilbert transform without performing the Hilbert transform DSP operations.

To generate a LSB OFDM signal occupying the $k$-th sub-wavelength spectral region, as seen in Fig. 1, the real part and (-1)-multiplied imaginary part of the produced complex OFDM signal $S_{k l}(t)$ are first up-sampled and then digitally filtered using an orthogonal digital shaping filter pair [4]. The produced LSB OFDM signal at the $k$-th sub-wavelength spectral region can be express as,

$$
\begin{gathered}
S_{L S B-k}(t)=M \uparrow\left\{\operatorname{Re}\left(S_{k 1}(t)\right)\right\} \otimes h_{k}^{I}(t)- \\
M \uparrow\left\{-\operatorname{Im}\left(S_{k 1}(t)\right)\right\} \otimes h_{k}^{Q}(t)
\end{gathered}
$$

where $M \uparrow$ represents the digital-domain up-sampling operation by a factor of $M .\left\{h_{k}^{I}(t), h_{k}^{Q}(t)\right\}$ stand for the $k$-th pair of the orthogonal digital shaping filters with I/Q indicating the in-phase/quadrature-phase digital filter type [4].

Very similar to the above-described digitally-filtered LSB signal generation process, the generation of the $k$-th digitally-filtered USB OFDM signal can also be written asgiven by,

$$
\begin{gathered}
S_{U S B-k}(t)=M \uparrow\left\{\operatorname{Re}\left(S_{k 2}(t)\right)\right\} \otimes h_{k}^{I}(t)+ \\
M \uparrow\left\{-\operatorname{Im}\left(S_{k 2}(t)\right)\right\} \otimes h_{k}^{Q}(t)
\end{gathered}
$$

where $S_{k 2}(t)$ is the complex OFDM signal which locates at the USB spectral region of the $k$-th sub-wavelength after the digital filtering operation.

After digitally combining the $k$-th LSB signal with the $k$-th USB signal together, the signal occupying the $k$-th sub-wavelength spectral region can be written as,
Formatted: Underline

Formatted: Underline

Formatted: Underline 


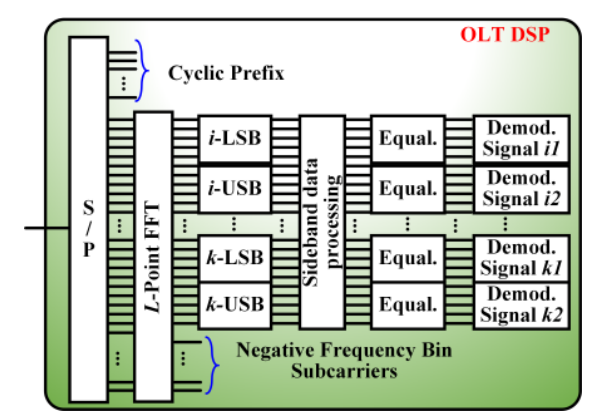

Fig. 3 A single FFT operation-enabled signal demultiplexing and recovery process in the OLT DSP. Equal.: equalization. Demod.: demodulation.

$$
\begin{aligned}
S_{\text {subwave-k }}(t) & =S_{\text {LSB-k}}(t)+S_{\text {USB-k}}(t) \\
& =M \uparrow\left\{\operatorname{Re}\left(S_{k 1}(t)\right)+\operatorname{Re}\left(S_{k 2}(t)\right)\right\} \otimes h_{k}^{I}(t)+ \\
M & \uparrow\left\{-\operatorname{Im}\left(S_{k 1}(t)\right)+\operatorname{Im}\left(S_{k 2}(t)\right)\right\} \otimes h_{k}^{Q}(t)
\end{aligned}
$$

Eq. (5) implies that the signal generation process of $S_{\text {subwave-k }}(t)$, which contains two different digitally-filtered SSB OFDM signals, can be simplified by just performing two similar digital filtering processes only. Such a simplified sub-wavelength signal generation process is also illustrated in Fig. 1, in which two SSB OFDM signals at the $i$-th sub-wavelength are simultaneously produced.

After a DAC and an optical intensity modulator, an optical signal produced by the $r$-th ONU can be expressed as,

$$
S_{o p t}(t)=\sigma_{r}(t) e^{j \beta_{r}(t)} \sqrt{1+\zeta_{r} * S_{r}(t)} e^{j 2 \pi f_{o p t} t}
$$

where $\zeta_{r}$ is the modulation index and $f_{\text {opt }}$ denotes the optical carrier central frequency. $\sigma_{r}(t)$ and $\beta_{r}(t)$ take into account the optical amplitude and phase related to the for theoptical intensity modulator. modulated optical signal. $S_{r}(t)$ stands for the electrical signal which can contain either a single SSB OFDM signal (a LSB OFDM signal or an USB OFDM signal) or two different SSB OFDM signals occupying the $r$-th sub-wavelength spectral region.

For the hybrid SSB OFDM-DFMA PON upstream application scenarios, the passively coupled optical signal from a total number of $N$ ONUs is given by,

$$
S_{N-o p t}(t)=\sum_{r=1}^{N}\left[\sigma_{r}(t) e^{j \beta_{r}(t)} \sqrt{1+\zeta_{r} * S_{r}(t)}\right] e^{j 2 \pi f_{o p t} t}
$$

To simplify the analytical derivation procedure, linear transmission systems are assumed in this section only. The impacts of nonlinear effects associated with both optical signal modulation/detection and optical signal transmissions over the considered IMDD PON systems are extensively investigated numerically in Sections III $\sim$ V of the paper. Under such a the linear transmission system assumption, in the OLT, after optical-electrical (O-E) conversion and an ADC, the received digital signal consisting of $U$ pairs of the LSB and USB OFDM signals is described as,

$$
S_{r x}(t)=\sum_{g=1}^{U}\left[S_{L S B-g}(t)+S_{U S B-g}(t)\right]
$$

To recover the received multiple SSB OFDM signals in the OLT, the adopted digital-domain signal demultiplexing and demodulation processes are illustrated presented in Fig. 3, in which similar to the previously-reported hybrid DSB OFDM-DFMA PONs [7], a single $L$-point FFT operation is applied with $L=2 B^{*} W$, here $W$ stands for the maximum number of digital shaping filter pairs. After the FFT operation, to identify the subcarriers for each SSB OFDM signal, the $L / 2$ subcarriers in the positive frequency bin are classified into $W$ groups each containing $B$ subcarriers. The subcarriers in the different groups locate at different sub-wavelength spectral regions, $A$ as such, the first $B / 2$ subcarriers in the $i$-th group correspond to the $i$-th LSB signal, while the remaining $B / 2$ subcarriers of the same subcarrier group correspond to the $i$-th USB signal. After having identified the LSB signal and the USB signal in each sub-wavelength, the sideband data processing is then applied, in which the subcarriers in all the LSB signals require a conjugation operation and a subcarrier reverse ordering operation, as illustrated in Fig. 3. While for the USB signals, no extra DSP operations are required before the OFDM equalization process. Similar to the reported hybrid DSB OFDM-DFMA PONs [7], the conventional OFDM signal equalization process is still applicable for recovering both the LSB and USB signals recon architecture.

Based on the above theoretical model, it is easy to understand that in the OLT, after the FFT operation, the subcarriers in the negative frequency bin can also be considered for recovering the SSB OFDM signals without compromising the transmission performances. In this paper, for simplicity, the subcarriers in the positive frequency bin are used in exploring the upstream performances of the proposed PON.

From the practical application point of view, in generating the digitally-_filtered SSB OFDM signals, the Hilbert-pair approach [4] can be utilized for constructing the required orthogonal digital shaping filter pairs. The impacts of the non-ideal digital shaping filters on the upstream performances are extensively explored in Section III V. In the ONUs and the OLT, the digital filtering process and the FFT operation as well as the subcarrier identification process are dynamically controllable by the embedded DSP controllers, as illustrated in Fig. 1. Therefore, dynamic and elastic connections can be established between the OLT and the ONUs through the centralized SDN controllers, which work together with their embedded DSP controllers to dynamically set the digital shaping filter pair coefficients and to identify the corresponding subcarriers after the FFT operation.

\section{UPSTREAM OPTIMUM ONU OPERATING CONDITIONS}

In this paper, to explore the hybrid SSB OFDM-DFMA PON performances and further demonstrate the advantages associated with the proposed PON, extensive upstream
Formatted: Underline

Formatted: Underline 
TABLE I

\begin{tabular}{|c|c|c|c|}
\hline \hline PARAMETER & \multicolumn{1}{c}{ VALUE } & \multicolumn{1}{c}{ PARAMETER } & \multicolumn{1}{c|}{ VALUE } \\
\hline DAC/ADC & $\begin{array}{c}30 \mathrm{GS} / \mathrm{s} \\
\text { @8-bit }\end{array}$ & $\begin{array}{c}\text { Digital-domain } \\
\text { Up-sampling Factor }\end{array}$ & $\mathrm{M}=8$ \\
\hline $\begin{array}{c}\text { Digital } \\
\text { Filter Length }\end{array}$ & $\mathrm{L}=64$ & $\begin{array}{c}\text { Clipping Ratio } \\
\text { (DSB case/SSB } \\
\text { case-I/II) }\end{array}$ & $\begin{array}{c}11 \mathrm{~dB} \\
9 \mathrm{~dB} \\
8 \mathrm{~dB}\end{array}$ \\
\hline $\begin{array}{c}\text { OFDM } \\
\text { IFFT/FFT Size }\end{array}$ & $32 / 256$ & Cyclic Prefix & $12.5 \%$ \\
\hline $\begin{array}{c}\text { OFDM Subcarrier } \\
\text { Modulation Format }\end{array}$ & $16-\mathrm{QAM}$ & $\begin{array}{c}\text { Data-bearing } \\
\text { Subcarriers of Each } \\
\text { OFDM Signal }\end{array}$ & 15 \\
\hline $\begin{array}{c}\text { Channel Bitrate for } \\
\text { SSB case I/II }\end{array}$ & $\begin{array}{c}6.25 \\
\mathrm{Gbit} / \mathrm{s}\end{array}$ & $\begin{array}{c}\text { Channel Bitrate for } \\
\text { DSB case }\end{array}$ & $\begin{array}{c}6.25 \\
\mathrm{Gbit} / \mathrm{s}\end{array}$ \\
\hline $\begin{array}{c}\text { PIN Detector } \\
\text { Sensitivity }\end{array}$ & $-17 \mathrm{dBm}$ & $\begin{array}{c}\text { PIN Detector } \\
\text { Quantum Efficiencies }\end{array}$ & 0.8 \\
\hline $\begin{array}{c}\text { Fiber Dispersion } \\
\mathrm{ps} / \mathrm{nm} / \mathrm{km}\end{array}$ & $\begin{array}{c}\text { Fiber } \\
\text { Dispersion Slope }\end{array}$ & $\begin{array}{c}0.08 \\
\mathrm{ps} / \mathrm{nm} \mathrm{m}^{2} / \mathrm{km}\end{array}$ \\
\hline $\begin{array}{c}\text { Fiber Kerr } \\
\text { Coefficient }\end{array}$ & $\begin{array}{c}2.6 \mathrm{e}-20 \\
\mathrm{~m}^{2} / \mathrm{W}\end{array}$ & $\begin{array}{c}\text { Fiber Loss } \\
\mathrm{dB} / \mathrm{km}\end{array}$ \\
\hline
\end{tabular}

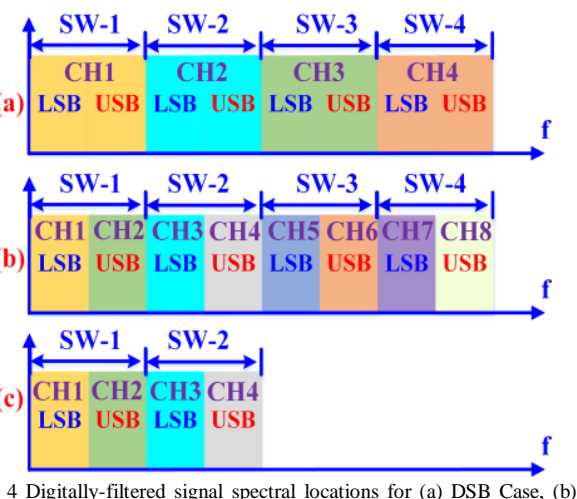
SSB Case-I and (c) SSB Case-II. SW: sub-wavelength. CH: channel.

transmission performance comparisons are made between the proposed PON systems and the previously-reported hybrid DSB OFDM-DFMA PON systems. To enable all these IMDD PONs to operate at their optimum conditions, it is vital to not only explore the PAPRs of the digitally filtered OFDM signals but also identify their corresponding optimum ONU operating conditions. In addition, for maximising the transmission performances of the hybrid DSB OFDM-DFMA PONs, whose performances are regarded as benchmarks for the proposed technique, it is of importance to identify the optimum sideband in each sub-wavelength.

\section{A. Simulation Models and Key Parameters.}

A representative IMDD PON with its architecture identical to that illustrated in Fig. 1 is considered in upstream numerical simulations. The PON consists of four ONUs each producing an optical signal occupying different DSB/SSB spectral bands. For performance comparisons, here three different cases are considered, including SSB Case-I, SSB Case-II and DSB Case. As shown in Fig. 4, in SSB Case-I, by making use of the
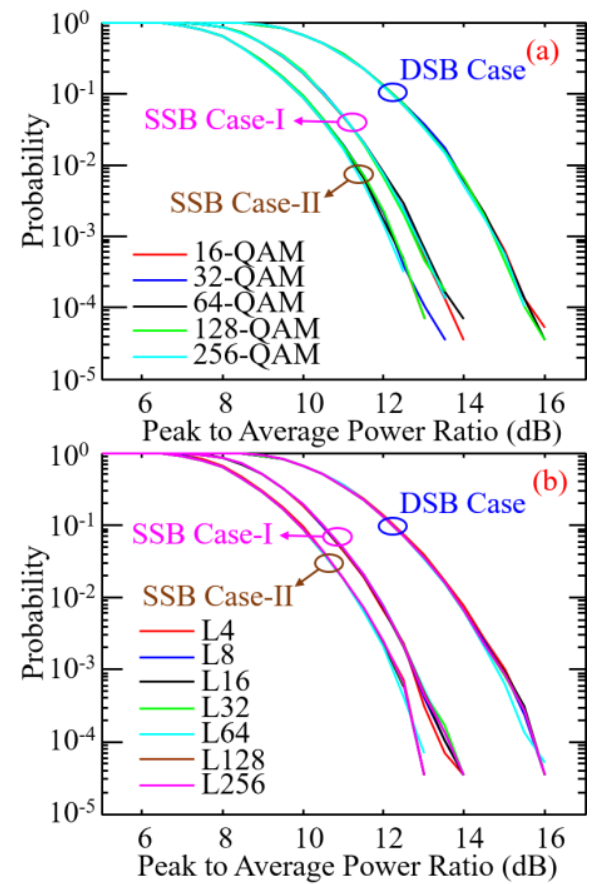

Fig. 5 CCDF curves of the digitally-filtered signals for (a) various modulation formats and adopting a fixed digital filter length of $\mathrm{L}=64$ and (b) various digital filter lengths and utilizing 16-QAM modulation format.

simplified digital filtering process shown demonstrated in Fig. 1 , each ONU produces a sub-wavelength signal simultaneously occupying the LSB and USB two different SSB spectral regionsbands. While in SSB Case-II, each ONU only generates a single SSB spectral band. For DSB Case, in each ONU, a single DSB spectral sub-band signal is produced after performing the digital filtering operation.

For DSB Case, a real-valued OFDM signal containing 15 data-bearing subcarriers is produced by the Hermitian symmetry in each ONU. While for these two SSB cases, complex OFDM signals each containing 15 data-bearing subcarriers are produced utilizing the complex OFDM signal generation process illustrated in Fig. 2. The main parameters adopted for generating the required real-valued and complex OFDM signals are listed in Table I.

To perform the digital filtering process, four orthogonal digital filter pairs with zero excess of bandwidth are produced by making use of the Hilbert-pair approach [4]. The central frequency of each orthogonal digital filter pair is defined as,

$$
f_{c i}=(2 \times i-1) f_{D A C / A D C} /(2 M), i=1,2,3,4
$$

where $f_{D A C / A D C}$ is the DAC/ADC sampling speed. For DSB Case, only in-phase digital shaping filters are utilized to digitally filter the up-sampled real-valued OFDM signals. While for 

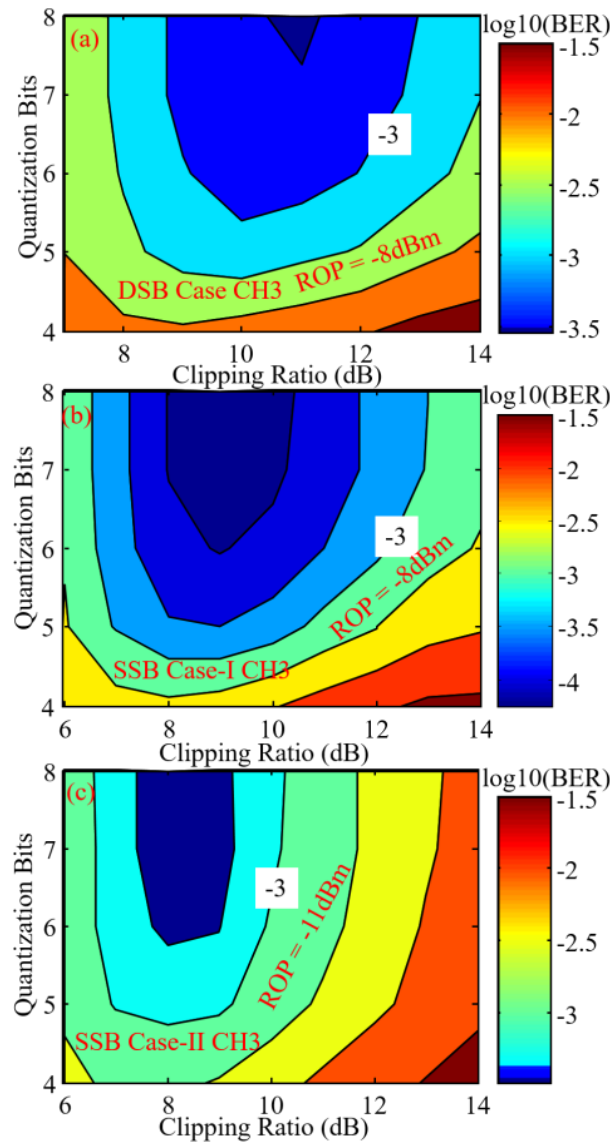

Fig. $6 \mathrm{CH}-3$ BER contour versus quantization bit resolution and clipping ratio. (a) DSB Case, (b) SSB Case-I and (c) SSB Case-II.

SSB Case-I and SSB Case-II, both the in-phase digital filters and the quadrature-phase digital filters are employed and their corresponding digital filtering processes are explicitly illustrated in Fig. 1. According to the theoretical analysis presented in [4], the minimum required $\mathrm{M}$ parameter value should be equal to the total number of signal channels considered in a targeted scenario, thus $\mathrm{M}=8$ is chosen throughout the paper. After conducting applying the digital filtering process, the signal spectral locations of the considered three cases are shown in Fig. 4, where - SSB Case-I and DSB Case have identical overall signal transmission bandwidths, but the channel number supported by SSB Case-I is doubled. While for SSB Case-II, its corresponding total channel number is identical to DSB Case, but it requires a halved overall spectral region.

For performing the E-O conversion in each ONU, similar to

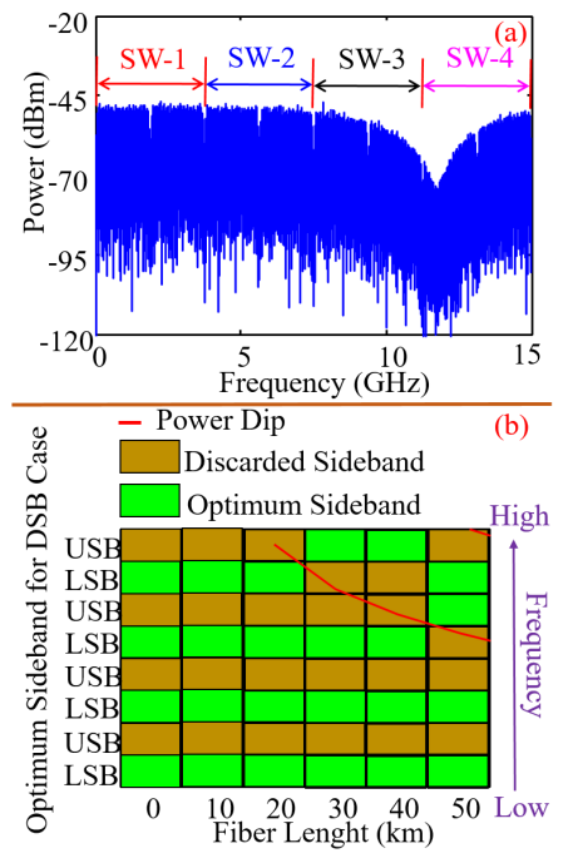

Fig. 7 Fiber transmission distance-dependent optimum sideband distributions for the hybrid DSB OFDM-DFMA PONs utilizing digital filtering lengths of $\mathrm{L}=64$. (a) signal spectrum after transmitting over $30 \mathrm{~km}$ SSMF. (b) optimum sideband distribution map.

the treatment in [7], an ideal optical intensity modulator is utilized, which can completely eliminate the optical beating interference (OBI) effect [11]. For upstream transmissions in practical IMDD PONs, to effectively minimizing the OBI effects, various different-ONUs can adopt different optical wavelengths and the minimum wavelength spacing between adjacent ONUs can be experimentally identified by utilizing the approach presented in [12]. For the considered three cases and taking into account the parameters listed in Table I, each DSB/SSB channel has an identical signal bitrate of $6.25 \mathrm{Gbit} / \mathrm{s}$. Since DSB Case and SSB Case-II support 4 channels, thus giving rise to an aggregated signal bitrate of $25 \mathrm{Gbit} / \mathrm{s}$. While for SSB Case-I, the supported 8 channels result in an aggregated signal bitrate of 50Gbit/s. In addition, in the OLT, an optical attenuator is employed to adjust the optical powers injected into the optical detector.

\section{B. PAPR of Digitally-filtered OFDM Signals}

Utilizing the parameters listed in Table I and the above-stated signal generation procedure, the PAPRs of the digitally- filtered signals are simulated for the considered three cases. In each case, similar PAPRs can be observed for all the digitally-filtered signals. Therefore, for DSB Case and SSB Case-II, the complementary cumulative distribution functions 
(CCDFs) of the digitally- filtered $\mathrm{CH}-1$ signals are depicted in Fig. 5, while for SSB Case-I, the digitally-_filtered $1^{\text {st }}$ sub-wavelength (SW-1) signal's CCDF curves are also plotted in the same figures. It can be seen in Fig. 5 that the proposed technique can reduce the PAPRs of the digitally-_filtered signals by $>2 \mathrm{~dB}$ at the probability of $1 \times 10^{-3}$. Such PAPR reductions are independent of the adopted digital filter characteristics and modulation formats as well as the signal spectral locations. In addition, compared with SSB Case-I, SSB Case-II has an extra $\sim 0.5 \mathrm{~dB}$ PAPR improvement because each digitally- filtered signal consists of a single SSB OFDM signal, while SSB Case-I contains two independent SSB OFDM signals. It is easy to understand that the reduced PAPRs can not only improve the system performance tolerance robustness to quantization noise induced by the DACs/ADCs with fixed resolution bits, but also relax the stringent requirements on linear dynamic operation ranges of the transceivers-embedded optical/electrical devices.

\section{Optimum Clipping Ratio and DAC/ADC Resolution Bits}

To enable the considered SSB/DSB cases to achieve their best performances, the optimum signal clipping ratios and DAC/ADC resolution bits are identified in optical back-to-back (B2B) systems for all the considered cases. The $\mathrm{CH}-3$ bit error fate (BER) contours versus resolution bits and clipping ratios are illustrated in Fig. 6, in obtaining which the received optical powers (ROPs) are set to be $-11 \mathrm{dBm}$ for SSB Case-II and $-8 \mathrm{dBm}$ for DSB Case and SSB Case-I. These different ROPs for the different cases are selected to not only ensure that BERs of $1 \times 10^{-3}$ are always achievable when utilizing 8-bit DAC/ADCs, but also clearly illustrate the impact of signal clipping on the BER performances. The results indicate that: 1) the proposed PON can reduce the DAC/ADC resolution bits by at least 1 bit for achieving BERs at forward error correction (FEC) limit of $1 \times 10^{-3} ; 2$ ) the identified optimum clipping ratios for DSB Case and SSB Case-I/II are $11 \mathrm{~dB}, 9 \mathrm{~dB}$ and $8 \mathrm{~dB}$ respectively. For these two SSB cases, their identified optimum clipping ratios are smaller than that identified in DSB Case due to the SSB-induced PAPR reductions. In the following sections, these identified optimum clipping ratios and 8-bit quantization are thus chosen to enable the ONUs to operate at their optimum conditions.

D. Optimum Sideband Selection for Hybrid DSB OFDM-DFMA PONs for Various Fiber Transmission Distances

As seen in Fig. 7(a), for 30km IMDD hybrid DSB OFDM-DFMA PONs, the signals locating at the $3^{\text {rd }}$ and $4^{\text {th }}$ sub-wavelength spectral regions suffer the severe channel fading effect. For achieving the best transmission performances, the LSB for $\mathrm{CH} 3$ and the USB for $\mathrm{CH} 4$ suffering the relatively less channel fading effect are thus selected. As a direct result of the transmission distance-dependent channel fading effect, the transmission distance-dependent optimum sideband distribution map is shown in Fig. 7(b), in obtaining which the optimum digital filter length of $\mathrm{L}=64$ identified in section $\operatorname{IV}(\mathrm{C})$ is utilized to minimize channel interferences. As
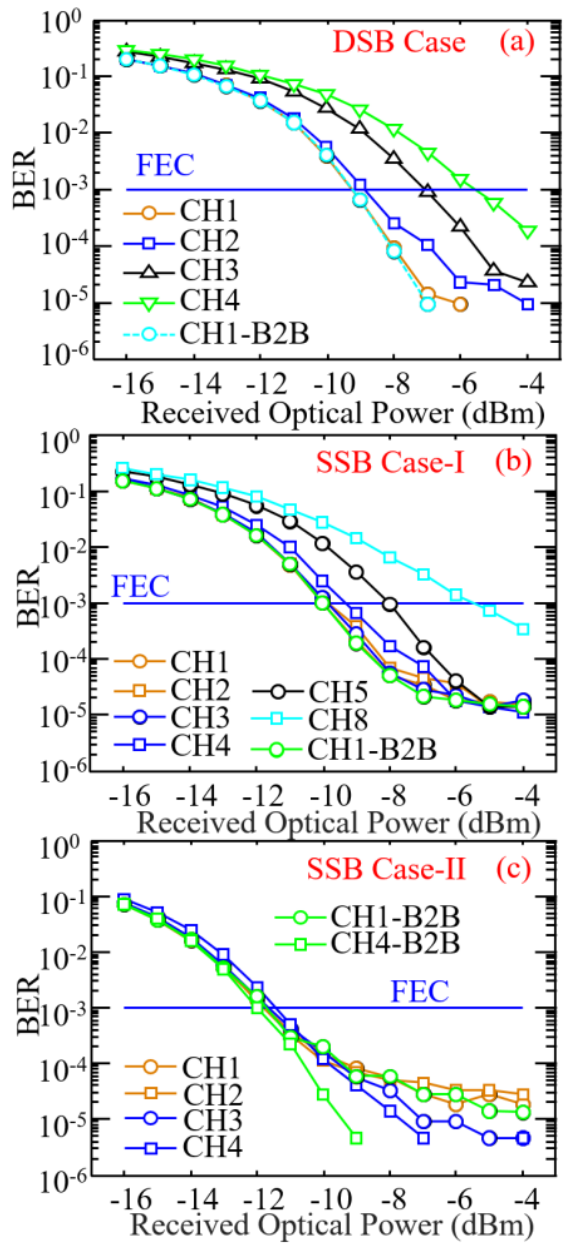

Fig. 8 30km SSMF transmission performances of (a) DSB Case, (b) SSB Case-I and (c) SSB Case-II.

seen in this figure, the optimum sidebands locate at both sides of the system frequency response dip lines caused by the channel fading effect. In addition, for DSB signals suffering the negligible channel fading effect, the LSB signal always has better transmission performance than the corresponding USB signal of the same sub-wavelength.

\section{UPSTREAM TRANSMISSION PERFORMANCE OF HYBRID SSB OFDM-DFMA PONS}

Utilizing the optimum ONU operating conditions identified in section III, the upstream transmission performances of the proposed IMDD hybrid SSB OFDM-DFMA PONs are extensively explored in this section. Spectral attention is 


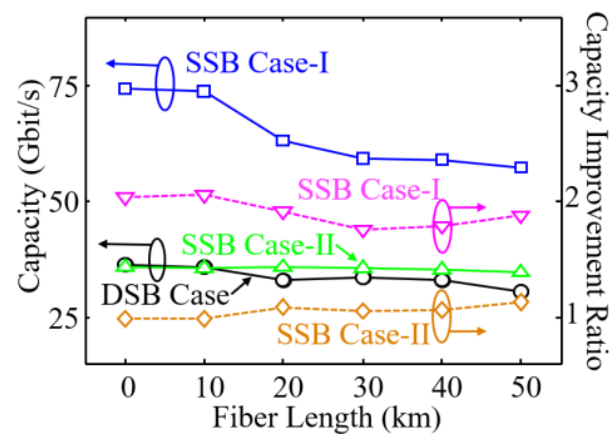

Fig. 9 Maximum aggregated upstream transmission capacity versus reach performances and corresponding SSB technique-induced upstream transmission capacity improvement ratio.

focused on the maximum aggregated upstream signal transmission capacity versus reach performances and system robustness against variations in digital filter characteristics. To highlight the advantages associated with the proposed PONs, the upstream transmission performances of the IMDD hybrid DSB OFDM-DFMA PONs are also computed, which are treated as benchmarks.

\section{A. Upstream Transmission Performances}

After $30 \mathrm{~km}$ standard single mode fiber (SSMF) transmission, the BER performances of all the involved channels for the considered three cases are illustrated in Fig. 8. The digital filter lengths are set to be $\mathrm{L}=64$ and the data-bearing sub-carriers of each involved OFDM signal are uniformly coded utilizing 16-QAM. In addition, the corresponding optical B2B upstream transmission performances of for $\mathrm{CH} 1$ are also plotted in the figure for performance comparison.

In Fig. 8(a), it can be found that DSB Case supports 4 usable channels with their BERs capable of reaching FEC limit. While for SSB Case-I, since its $\mathrm{CH} 6$ and $\mathrm{CH} 7$ are not operational due to the channel fading effect, it can thus support a total of 6 usable channels, as seen in Fig. 8(b). It implies that the proposed SSB technique can improve the spectral efficiency by a factor of 1.5 when utilizing an identical signal modulation format across all subcarriers. If adaptive bit-loading is adopted for all involved channels, the maximum aggregated upstream capacity can be almost doubled by the proposed technique, as seen in Fig. 9.

For SSB Case-II, all the channels suffer the negligible channel fading effect and thus have similar transmission performances. It can also be found that DSB Case and SSB Case-II support the same number of identical useable channels. number. However, SSB Case-II has $>2.5 \mathrm{~dB}$ power budget improvements due to the SSB-induced enhancements in effective optical signal to noise ratio (OSNR). Our numerical simulation results also show that such power budget improvements are independent of transmission distances and digital filter characteristics. In addition to the above-mentioned power budget improvements, the required signal transmission
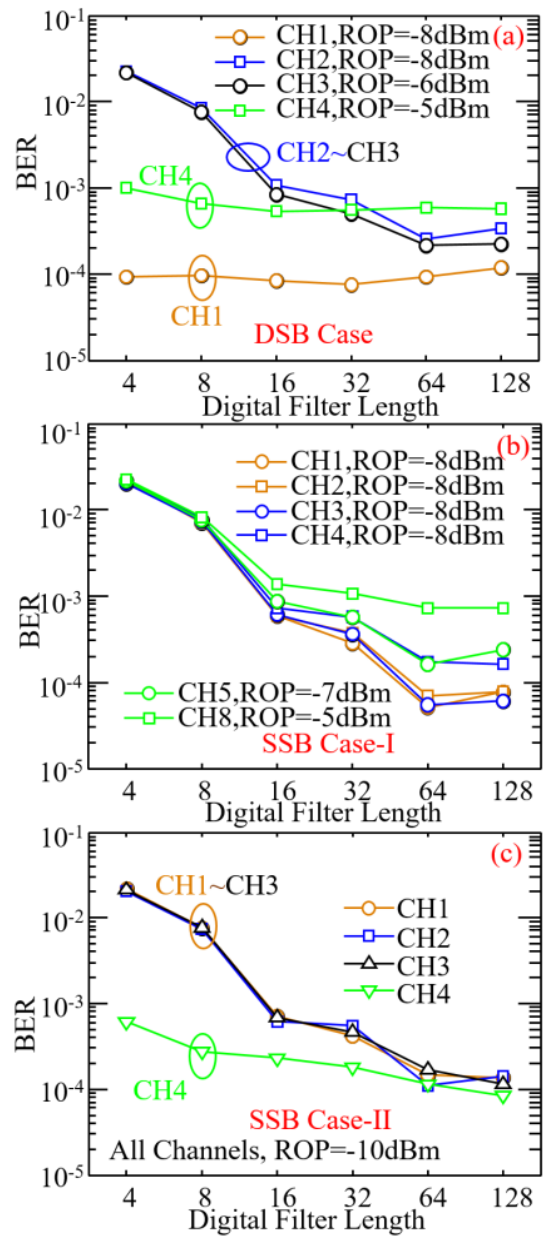

Fig. 10 Impacts of digital filter characteristics on upstream transmission performances. (a) DSB Case, (b) SSB Case-I and (c) SSB Case-II.

bandwidth for SSB Case-II is just half of that required by DSB Case, as seen in Fig. 4. Such reduced signal transmission bandwidth requirements allow the transceivers to use low-cost and low-bandwidth optical/electrical devices.

It is also worth mentioning the following two aspects: 1) the similar transmission performances of the $\mathrm{CH}-1$ signals between the $30 \mathrm{~km}$ transmission systems and the optical B2B systems indicate that for all the considered involved cases, the channels suffering negligible channel fading effect can have negligible performance degradations, and 2) for SSB Case-II, compared to other low frequency channels, $\mathrm{CH} 4$ has the better transmission performance, because it suffers less channel interferences as discussed in Section IV(C). 
B. Maximum Aggregated Upstream Transmission Capacity

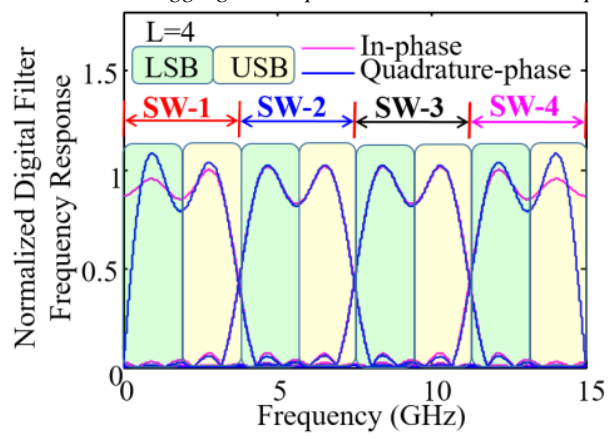

Fig. 11 Normalized in-phase and quadrature-phase digital filter frequency responses for the digital filter length of $\mathrm{L}=4$.

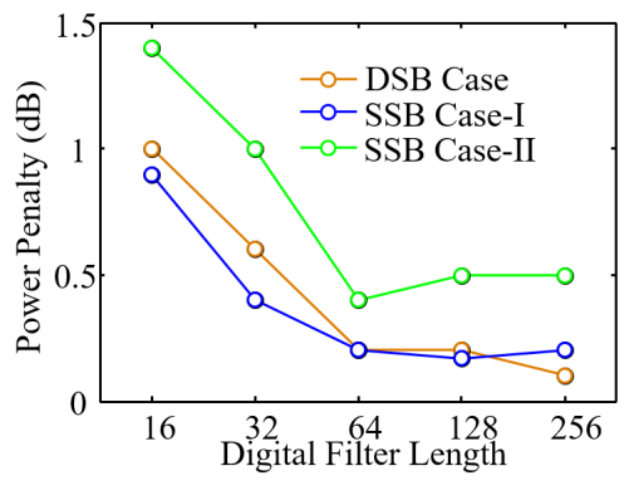

Fig. 12 Channel interference-induced power penalty for $30 \mathrm{~km}$ upstream signal transmissions.

\section{versus Reach Performances}

As seen in Fig. 8(a) and Fig. 8(b), the SSB technique considerably improves the spectral efficiency, which can give rise to an improvement in the maximum aggregated upstream transmission capacity. To verify this statement and further examine the effectiveness performance of the SSB technique in improving the upstream signal transmission capacity, adaptive bit-loading is utilized for each considered involved OFDM signal with signal modulation formats varying from 4/16/32/64-QAM to 128-QAM. The obtained maximum aggregated upstream signal transmission capacity versus reach performance for each considered case is depicted in Fig. 9. In addition, the SSB technique-induced capacity improvement ratios are also plotted in the figure. The ROPs of $-4 \mathrm{dBm}$ and the digital filter lengths of $\mathrm{L}=64$ are adopted. Comparing the results between SSB Case-I and DSB Case, it can be found that the upstream signal transmission capacity is almost doubled when the DSB case and the SSB case have similar signal transmission bandwidths. Moreover, the results between SSB Case-II and DSB Case are very similar, as these two cases have identical useable channel numbers. However, compared to DSB Case, the proposed SSB PON can not only halve the required overall signal transmission bandwidth for delivering the same upstream transmission capacity, but also give rise to $>2.5 \mathrm{~dB}$ power budget improvements as discussed in section IV(A).

C. Impacts of Digital Filter Characteristics on Upstream Transmission Performances

In the hybrid DSB (SSB) OFDM-DFMA PONs, digital filtering is essential in terms of allocating the DSB (SSB) OFDM signals at suitable sub-wavelengths. Generally speaking, a larger digital filter length can result in a better digital filtering performance, but inevitably increases the digital filter DSP complexity [7]. It is therefore vital to explore the impacts of the digital filter characteristics on the upstream transmission performance, based on which the minimum digital filter length can be identified for achieving the targeted performances for in a specific application scenario.

Fig. 10 presents the digital filter characteristic-induced impacts on the upstream BER performances for the considered three PON cases. The fiber transmission distance is $30 \mathrm{~km}$. For each considered involved OFDM channel, the 16-QAM signal subcarrier modulation format is employed for each subcarrier and the ROPs are chosen in the vicinity of their corresponding receiver sensitivities. It can be found that for all the considered cases, the minimum digital filter lengths are $\mathrm{L}=64$, corresponding to which digital filtering-induced performance degradations are negligible.

In addition, it is also shown in Fig. 10 that the BER performances of both $\mathrm{CH} 1 / \mathrm{CH} 4$ for DSB Case and $\mathrm{CH} 4$ for SSB Case-II are almost independent of digital filter length, this is because these channels suffer negligible power leakages arising from the overlapped digital filter frequency responses. everlapping. This can be understood by considering Fig. 11, where normalized in-phase and quadrature-phase digital filter frequency responses with forfixed digital filter lengths of $\mathrm{L}=4$ are plotted for different sub-wavelengths. It is easy to find in Fig. 11 that, for DSB Case, the digital filter frequency response overlapping-induced power leakage mainly occurs at the CH1's USB spectral region and CH4's LSB spectral region, both of which are, however, not chosen to convey the any information. As such, the digital filter length-independent performances are thus observed for these two channels. While for the SSB case, both the in-phase digital filters and the quadrature-phase digital filters are utilized to generate the SSB OFDM signals at different sub-wavelengths. Therefore, in addition to aforementioned digital filter frequency response overlapping, the digital filter frequency response ripples can also contribute to the power leakages between the two channels of the same sub-wavelength. As such, for SSB Case-I, all the involved OFDM channels have similar BER developing trends as shown in Fig. 10(b). However, for SSB Case-II, due to its location in the highest spectral region, the $4^{\text {th }}$ channel suffers negligible digital filter frequency response overlapping-induced power leakages, thus leading to a different BER developing trend compared to other channels.

To further examine the abovementioned physical 
mechanisms behind the digital filter length associated signal distortions, and more importantly, to verify the identified minimum digital filter length value from a different angle, the digital filter length-dependent channel interference-induced power penalties for all the considered three cases are plotted in Fig. 12, where the power penalty is defined as the difference of ROPs corresponding to the FEC limit with and without a sub-wavelength-wide spectral space between two adjacent ONUs. In numerically simulating Fig. 12, the 16-QAM modulation format is used for each involved OFDM signal. The $30 \mathrm{~km}$ SSMF transmission distance is considered.

It is shown in Fig. 12 that for digital filter lengths of $>\mathrm{L}=>64$, the power penalty curves become flattened, indicating that $\mathrm{L}=64$ is indeed the minimum digital filter lengths for all the considered cases. Since the minimum digital filter length is independent of ONU count [13], it is therefore easy to understand that the digital filter length-dependent power penalty behaviours presented in Fig. 12 are applicable regardless of ONU counts.

In addition, the results in Fig. 12 also indicate that, in comparison with SSB Case-I, SSB Case-II suffers higher power penalties across the entire digital filter length variation range, this is because for SSB Case-II, the introduced spectral spaces can not only reduce the channel interferences between neighboring sub-wavelength channels but also minimize the channel interferences between the channels of the same sub-wavelength. While for SSB Case-I, the introduced ONU spectral spaces can only reduce the channel interferences between the channels within the adjacent sub-wavelengths.

\section{DifFERENTIAL ONU Optical LaUnch Power DyNamic RANGE}

Utilizing the simulation conditions identical to those adopted in computing Fig. 12 and employing the identified minimum digital filter length of $\mathrm{L}=64$, the system robustness against differential ONU optical launch power [7] for the considered three cases are explored in Fig. 13. The ROP is always fixed at $-4 \mathrm{dBm}$. The simulated differential optical launch power dynamic ranges for all the considered ONUs before and after 30km upstream transmissions are presented in Fig. 13.

As seen in Fig. 13(b), after transmitting through $30 \mathrm{~km}$ SSMFs, $<2 \mathrm{~dB}$ differences in differential ONU optical launch power dynamic range between DSB Case and SSB Case-I can be achievable, indicating that the SSB Case-I-enabled improvement in upstream signal transmission capacity does not considerably affect the differential ONU optical launch power dynamic range. While for the hybrid SSB OFDM-DFMA PONs delivering upstream signal transmission capacities identical to those for the hybrid DSB OFDM-DFMA PONs, the ONUs locating at the high frequency spectral region improve their differential optical launch power dynamic ranges by $>1.2 \mathrm{~dB}$. Such an improvement is mainly because of the SSB-induced enhancements in system robustness against the channel fading effect. This is evidenced by the fact that negligible differences in ONU differential optical launch power dynamic range are observed for SSB Case-II before and after $30 \mathrm{~km}$ SSMF transmissions.
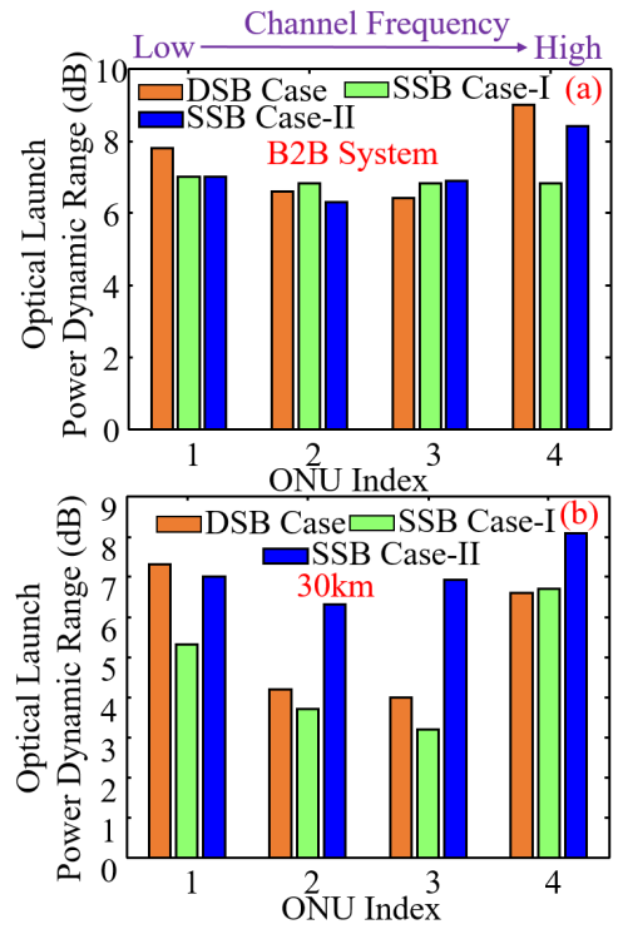

Fig. 13 Differential ONU optical launch power dynamic ranges for (a) optical B2B system and (b) 30km SSMF transmission.

\section{CONCLUSIONS}

A novel IMDD-based hybrid SSB OFDM-DFMA PON has been proposed and extensively investigated, for the first time, in which multiple independent SSB OFDM channels are multiplexed utilizing the ONU-embedded software-configurable orthogonal digital filters, and simultaneously demultiplexed and demodulated by a single FFT operation in the OLT without utilizing matching filters. In generating and demodulating the multiple SSB OFDM signals, the Hilbert transform DSP operations are not applied in both the ONUs and the OLT.

The upstream optimum ONU operating conditions have been identified for both the proposed hybrid SSB OFDM-DFMA PONs and the previously-reported hybrid DSB OFDM-DFMA PONs. It has been shown that compared to the DSB case, the proposed SSB PON can lead to $>2 \mathrm{~dB}$ reductions in PAPRs of digitally-_filtered OFDM signals. Such PAPR reductions are independent of digital filter characteristics, modulation formats and signal spectral location. As a direct result of the reduced PAPRs, $>2 \mathrm{~dB}$ reductions in optimum signal clipping ratios are also observed, which decrease the minimum DAC/ADC resolution bits by $>1$-bit for achieving BERs of at $1 \times 10^{-3}$. 
By utilizing the identified optimum ONU operating conditions, extensive upstream transmission performance comparisons have been made between the aforementioned two hybrid OFDM-DFMA PONs. Numerical simulations have indicated that the proposed hybrid SSB OFDM-DFMA PONs still maintain all the advantages associated with the hybrid DSB OFDM-DFMA PONs. More importantly, for IMDD PONs with identical overall signal transmission bandwidths, in comparison with the DSB PON, the proposed SSB PON can double the maximum aggregated upstream transmission capacity without considerably compromising their differential ONU optical launch power dynamic ranges. While for the IMDD PONs delivering similar aggregated upstream transmission capacities, in comparison with the DSB PON, the proposed SSB PON offers extra unique features including: 1) a halved overall system bandwidth, 2) $>2.5 \mathrm{~dB}$ upstream system power budget improvements, and 3) $>1.2 \mathrm{~dB}$ enhancements in differential optical launch power dynamic range for ONUs locating at high frequency spectral regions.

To rigorously verify the proposed hybrid SSB OFDM-DFMA PON architecture presented in this paper and further evaluate the feasibility of utilizing the proposed PON to seamlessly converge optical access networks and mobile front-haul/back-haul networks, experimental investigations including real-time experimental demonstrations are currently being undertaken in our research lab, by making full use of our -real-time DFMA PON experimental demonstrations incorporating commercially available low-cost optical/electrical components [13]. In comparison with the DFMA PONs, the overall OLT hardware and software complexity is lower for the proposed PONs. and corresponding Detailed research results will be reported elsewhere in due course.

\section{REFERENCES}

[1] P. T. Dat, A. Kanno, N. Yamamoto, and T. Kawanishi, "Seamless convergence of fiber and wireless systems for 5G and beyond networks," J. Light. Technol., vol. 37, no. 2, pp. 592-605, Jan. 2019

[2] K. Sone, G. Nakagawa, Y. Hirose, and T. Hoshida, "Demonstration of simultaneous multiple ONUs activation in WDM-PON system for $5 \mathrm{G}$ fronthaul," Proc. Opt. Fiber Commun., San Diego, CA, USA, 2018, Paper M2B.6.

[3] J. Zhang, Y. Xiao, D. Song, L. Bai, and Y. Ji, "Joint wavelength, antenna, and radio resource block allocation for massive MIMO enabled beamforming in a TWDM-PON based fronthaul," J. Light. Technol., vol. beamforming in a TWDM-P. pp. 1396-1407, Feb. 2019.

[4] M. Bolea, R. P. Giddings, M. Bouich, C. Aupetit-Berthelemot, and J. M. Tang, "Digital filter multiple access PONs with DSP-enabled software reconfigurability," J. Opt. Commun. Netw., vol. 7, no. 4, pp. 215-222, Apr. 2015

[5] X. Duan, R. P. Giddings, S. Mansoor, and J. M. Tang, "Experimental demonstration of upstream transmission in digital filter multiple access PONs with real-time reconfigurable optical network units," $J$. Opt. Commun. Netw., vol. 9, no. 1, pp. 45-52, Jan. 2017.

[6] M. L. Deng, A. Sankoh, R. P. Giddings, and J. M. Tang, "Experimental demonstrations of $30 \mathrm{~Gb} / \mathrm{s} / \lambda$ digital orthogonal filtering-multiplexed multiple channel transmissions over IMDD PON systems utilizing 10G-class optical devices," Opt. Express, vol. 25, no. 20, pp. 24251-24261, Oct. 2017.

[7] Y. X. Dong, R. P. Giddings, and J. M. Tang, "Hybrid OFDM-digital filter multiple access PONs," J. Light. Technol., vol. 36, no. 23, pp. 5640-5649, Dec. 2018.
[8] Y. X. Dong, W. Jin, R. P. Giddings, M. O'Sullivan, A. Tipper, T. Durrant, and J. M. Tang, "Hybrid DFT-spread OFDM-digital filter multiple access PONs for converged 5G networks," J. Opt. Commun. Netw., vol. 11, no. 7, pp. 347-353, Jul. 2019

[9] L. Marple, "Computing the discrete-time "analytic" signal via FFT," IEEE Trans. Signal Processing, vol. 47, no. 9, pp. 2600-2603, Sep. 1999.

[10] Y. Zeng, Z. Dong, Y. Chen, X. Wu, H. He, J. You, and Q. Xiao, "A novel CAP-WDM-PON employing multi-band DFT-spread DMT signals based on optical Hilbert-transformed SSB modulation," IEEE Access, vol. 7, pp. 29397-29404, Mar. 2019.

[11] S. M. Jung, K. H. Mun, S. Y. Jung, and S. K. Han, "Optical-beat-induced multi-user-interference reduction in single wavelength OFDMA PON upstream multiple access systems with self-homodyne coherent detection," J. Light. Technol., vol. 34, no. 11, pp. 2804-2811, Jun. 2016.

[12]X. Q. Jin and J. M. Tang, "Experimental investigations of wavelength spacing and colorlessness of RSOA-based ONUs in real-time optical OFDMA PONs", J. Light. Technol., vol. 30, no. 16, pp. 2603-2609, Aug. 2012.

[13]E. Al-Rawachy, R. P. Giddings, and J. M. Tang, "Experimental demonstration of a real-time digital filter multiple access PON with low complexity DSP-based interference cancellation," J. Light. Technol., vol.37, no. 17. pp. 4315-4329, Sept. 2019. early access, Jun. 2019
Formatted: Underline

Formatted: Underline

Formatted: Underline

Formatted: Underline
Formatted: Underline

Formatted: Underline

Formatted: Underline

Formatted: Underline

Formatted: Underline

Formatted: Small caps, Kern at $14 \mathrm{pt}$

Formatted: English (United Kingdom), Small caps, Kern at $14 \mathrm{pt}$ 